Voix et Images

volxetimages

\title{
L'esthétique du détail dans Nous parlerons comme on écrit
}

\section{Claudine Potvin}

Volume 14, numéro 1 (39), automne 1988

France Théoret : narratrice de la subjectivité

URI : https://id.erudit.org/iderudit/200751ar

DOI : https://doi.org/10.7202/200751ar

Aller au sommaire du numéro

Éditeur(s)

Université du Québec à Montréal

ISSN

0318-9201 (imprimé)

1705-933X (numérique)

Découvrir la revue

Citer cet article

Potvin, C. (1988). L'esthétique du détail dans Nous parlerons comme on écrit.

Voix et Images, 14(1), 39-49. https://doi.org/10.7202/200751ar d'utilisation que vous pouvez consulter en ligne.

https://apropos.erudit.org/fr/usagers/politique-dutilisation/ 


\title{
L'esthétique du détail dans Nous parlerons comme on écrit
}

\author{
par Claudine Potvin, Université d'Alberta, Edmonton
}

Ecrire par fragments: les fragments sont
alors des pierres sur le pourtour du cercle: je
m'étale en rond: tout mon petit univers en $_{\text {miettes; au centre, quoi? }}{ }^{1}$

Des poussières. Des miettes. Des détails. Des mots. Hors-texte. Des mots dont le sens fuit, glisse entre les doigts. Des histoires sans contexte: des histoires ordinaires, des histoires à dormir debout. Le texte s'étale de tout son long sur un pan de mur en briques, une cour d'école, une maison en ruines, une odeur de bière, un corps mutilé, morcelé, vidé, une voix sourde, fêlée, cassée, éteinte, une minuscule boule verte gardée à l' ail, tenue en main (p. 71) ${ }^{2}$. Une image au détour d'une phrase, un tableau mexicain, un flash inscrit dans la mémoire. Banalités du quotidien. Avant la composition, il y a donc l'implication des images, des détails, des scènes minuscules qui enveloppent des sens multiples, contiennent des objets innombrables, recèlent des figures inouies et résonnent de mille «feux de langage». ${ }^{3}$

C'est bien ce rapport au réel que le roman de France Théoret, Nous parlerons comme on écrit, questionne. L'auteure y affirme la nécessité impérieuse, lucide, de mettre en mots, de témoigner d'une existence rompue afin de survivre et de pouvoir [...] donner aux mots leur mesure afin qu' ils déroulent au plus près, au moins un jour, l'expression de l'existence (p. 107). Dire même si Les mots la brisent car elle tourne en rond dans ses mots et elle vit dans ça, vivante de paroles qui sont des paroles, vivante d'être traversée par la langue (p. 39).

Du réel à la (fausse?) représentation, France Théoret tisse un lieu décousu d'apparences piégées d'avance. Au centre du/des récit(s), le parcellaire, le morcellement, la dépossession d'une instance énonciatrice creuse, filtrée, niée: lente/ vaine appropriation de la parole des mères, douloureuse gestation d'une écriture qui s'inscrit sur le corps brisé des femmes, une voix entre le lourd silence des hommes et l'immensité et le désœuvrement des cris de l'autre. Piétinements, retours, passages impossibles. L'imaginaire enfermé puis découpé en pièces détachées. Dans une entrevue qu'elle accordait à Estuaire, l'auteure disait écrire

1 Roland Barthes par Roland Barthes, Paris, Seuil, 1975, p. 96.

2 France Theoret, Nous parlerons comme on écrit, Montréal, les Herbes rouges, 1982. Pour toute référence à ce texte, j'indiquerai la page entre parenthèses à la fin de la citation.

3 Reda Bensmaïa, «Du fragment au détail», Poétlque, n 47, septembre 1981, p. 368. 
une certaine manière de sentir le réel, une sensibilité qui s'ouvre à la réalité; consciente du geste d'écrire et consciente également du contenu, «du réel obsédant puisque L'un et l'autre ne fonctionnent pas en parallèle mais bien dans le croisement. ${ }^{4}$

Le roman de France Théoret se présente comme un corps roué de coups, une série de fragments accumulés en strates distinctes ${ }^{6}$, un corps morcelé sans cesse interrompu dans sa marche sans but précis, sans enchaînement. Interruptions, arrêts, pauses, reprises, répétitions, retours, variantes, hachures, équivoques marquent un texte barré qui tombe sous le coup des interdits, mais en cela même texte multiple, éclaté, refusant le sens absolu, le sens unique. Ainsi le texte semble-t-il toujours s' inscrire en marge, hors-cadre, à la limite de l'effritement des phrases et du temps et de l'éparpillement du corps. Écriture plurielle où se mêlent plusieurs discours. On l'a signalé à maintes reprises, le roman tient à la fois de l'autobiographie, du journal, des mémoires, de la poésie, de l'essai, du fragment, du récit anecdotique, etc., mélange des genres que France Théoret qualifie de métissage. Autant de registres textuels s'articulent autour d'éléments concrets, de faits, d'anecdotes d'une vie ou d'une partie de vie. Autour de détails en fait.

Le détail opère précisément en tant qu'élément de contenu minimal du récit. La narratrice dit entre[r] dans tous les détails (p. 140). En fait, elle n'en sort guère, le détail fonctionnant aussi comme un principe de composition préalable. La structure même du roman suggère le détaillisme, une forme de pointillisme même. Au sujet de sa méthode d'apprentissage en peinture, Barthes raconte:

$N$ ayant pratiqué en peinture que des barbouillages tachistes, je décide de commencer un apprentissage régulier et patient du dessin; [...] irrésistiblement, au lieu de chercher à représenter les proportions, l'organisation, la structure, je copie et j'enchaîne naïvement détail par détail; [...] En somme, je procède par addition, non par esquisse; j'ai le goût préalable (premier) du détail, du fragment, du rush, et l'inhabileté a le conduire vers une «composition»: je ne sais pas reproduire «les masses». ${ }^{7}$

France Théoret procède aussi par addition et par couches superposées. Dans Nous parlerons comme on écrit, la «composition» des «détails» constitue en soi le

4 Gérald Gaudet, «Une écriture responsable (entretien avec France Théoret)», Estuaire, $n^{\circ} 38,1986$, p. 110-111.

5 Renée-Berthe Drapeau, «Féminins singuliers. Pratiques d'écriture: Brossard, Théoret», mémoire de maîtrise, Université de Sherbrooke, 1985, p. 42.

6 Madeleine Ouellette-Michalska, «Vivre comme on écrit?», le Devoir, 15 mai 1982, p. 19.

7 Roland Barthes par Roland Barthes, op. cit., p. 97. 
récit enchaîné; rien d'autre ne doit absolument y conduire. De plus, l'organisation naît du désordre affiché.

Agencement de détails mis en jeu dans et par le texte, détails de l'œuvre d'art racontés, commentés en bas de page (ou à la suite ou en marge de) ou sous l'image dans un autre récit, dans une autre «histoire» de l'art. Sorte de récupération constante du texte ou de la toile morcelée: une baigneuse de Rubens, un corps de Gauguin, une forme de Moore, une scène de Ribera, une musique de Schönberg, Varèse ou Mahler, un extrait des sceurs Brontè, de Thérèse d'Avila, de Laure Conan, de Barthes, etc. Le détail ne semble alors représenter qu'une partie d'un tout, forçant et l'artiste et le récepteur (lecteur/amateur d'art/mélomane, etc.) à privilégier un rapport d'incomplétude, de renvoi, de manque à être.

Or, un détail, c'est bien sûr l'(in)signifiant, le futile, le petit, le minuscule, l'infime, le vide, ce qui n'est pas très important ou ce qui est dénué d'intérêt. Sa soustraction n'altère guère le sens du tableau ou du récit, semble-t-il. Cependant, dans un autre ordre d'idées, le détail, c'est parfois l'ajout qui change tout, l'élément de surenchère dans la décoration, l'omement, l'accessoire dans le système de la mode, voire l'excès, le surplus, le trop-plein. En tant qu'ornementation, le détail a longtemps représenté la décadence, la désintégration de l'harmonie classique en ce que l'ornement acquiert une certaine autonomie par rapport à la construction globale. Au niveau de l'écrit, l'utilisation systématique du détail «détaché» et du fragment tend à renforcer l'indépendance de la lettre, du mot, de la page. Lukács proposait de faire la différence entre les bons et les mauvais détails, soit entre les détails utiles dans la partie proprement descriptive de la diégèse (Balzac) et l'usage abusif du détail tel que pratiqué par le naturalisme ou le modernisme (Zola, Joyce ou Kafka par exemple) ${ }^{8}$. Paradoxalement, le détail échappe à l'œil dans sa fonction réductrice alors qu'il s'assimile au trompe-l'œil ou au tape-à l'œil dans sa fonction de surenchère. Là on le remarque à peine puisqu'il exige une observation minutieuse pour être repéré; la place qu'il occupe semble presque (in)sensée. Ici il saute aux yeux; surcharge de sens, inflation par rapport à l'ensemble.

Une longue tradition néo-classique situe le détail en fonction de deux catégories esthétiques: l'idéal, conçu à partir d'une idée absolue du beau ou de la perfection, argument d'ordre qualitatif; le sublime, qui reprend les notions d'équilibre, de proportion juste, d'élégance, élément d'ordre quantitatif'. Dans les deux cas, il va de soi qu'il s'agit de l'établissement d'une norme que le détail (voire l'importance du détail, la profusion des détails, etc.) risque à tout moment de transgresser, menaçant de la sorte l'unité de l'œuvre. Le détail ne se justifierait donc que par un équilibre soigneusement maintenu entre les parties et le tout, ce qui confirmerait la grandeur de l'œuvre. Ce sera encore la position hégélienne qui, poussée à la limite, aboutit à ce que Naomi Schor appelle la sublimation du détail, soit à la remise en question de la notion même d'insignifiance-vulgarité du

8 Voir Naomi Schor, Reading in Detall. Aesthetics and the Feminine, New York et Londres, Methuen, 1987. Voir le chapitre 3.

Ibid., p. 15. 
détail. Elle ajoute que, selon cette esthétique du néo-classicisme, In order for the «sum on insignificance» which constitute the décor of everyday life to have access to the world of art, they must acquire «the look of independance and total life and freedom which lies at the root of the essence of beauty.$^{10}$ Autant dire disparaître en tant que détail.

Nous parlerons comme on écrit fait précisément éclater cette vision d'une œuvre globale dans laquelle chaque partie contribuerait à créer une dimension unique, absolue, parfaite. Suzanne Lamy remarque à juste titre que le détail renvoie à l'ensemble ${ }^{11}$; certes, mais le détail ne le contient pas, pas plus qu'il $n$ 'y conduit. En ce sens, la tentative de montrer la connexion entre les différents fragments du roman dans le but d'en dégager une cohérence sémantique ou autre, débouche sur une fausse piste ${ }^{12}$. Le texte/puzzle ne ferait ainsi sens que considéré dans son ensemble; l'écriture détailliste ou l'écriture fragmentée permet d'en douter. Il y a des glissements révélateurs, bien sûr, de mots à mots, d'images et de thèmes entre eux, mais faut-il les relier à tout prix à un tout, idéal et soi-disant esthétiquement nécessaire. Ce serait se limiter à une conception du détail utilitaire, purement fonctionnel, qui aurait pour rôle principal d'associer des éléments entre eux, de mettre à jour des idées, de rendre évidente une certaine pratique herméneutique du texte ${ }^{13}$.

Si le détail signifiant menace, c'est qu'il ébranle le savoir dans sa miniaturisation et qu'il a tendance à pervertir l'ordre hiérarchique de l'œuvre d'art which clearly subordinates the periphery to the center, the accessory to the principal, the foreground to the background. ${ }^{14}$ Le détail force le récit à une position de décentrement, de marginalité. Dans Nous parlerons comme on écrit, le discours fonctionne par cercles qui rejettent constamment le lecteur à l'extérieur du récit premier ou précédent. Les différentes parties tendent à s'autonomiser, la narratrice tourne en rond d'une page à l'autre, propulsée par une force centrifuge qui projette le corps textuel/sexuel d'une femme non re/liée sur les murs de son angoisse. Le texte de France Théoret comme l'écriture de/en détail(s) interroge les principes mêmes d'unité et de cohérence, interrogation formulée à tous les niveaux (grammatical, stylistique, sémantique, narratif, etc.). In illo tempore, écrit France Théoret, ainsi commence ce qui n'a de cesse de recommencer. La nostalgie porte sur le forçage d' enchaîner de cause deffet, la fixation d' une certitude. Je pense et raye l' association entre je et pense. Je n' ai plus jamais eu le même rapport à l'écriture après (p. 13).

$10 \quad$ Ibid., p. 36-37.

11 Suzanne Lamy, «Des résonnances de la petite phrase: "Je suis un nceud" de France Théoret», dans Féminité, Subversion, Ecriture, Montréal, les Éditions du Remue-ménage, 1983 , p. 139.

12 Voir, par exemple, l'entreprise de Gisèle Tuaillon dans sa thèse, «France Théoret ou la parole empêchée», D.E.A., Université de Franche-Comté, Besançon, France, 1985.

13 Voir à ce sujet l'article de Horst S. Daemmrich, \&The Aesthetic Function of Detail and Silhouette in Literary Genres», Yearbook of Comparative Criticism 3, Theories of Literary Genre, édité par Joseph P. Strelka, University Park: Penn State University Press, 1978, p. 112-122.

14 Naomi Schor, op. cit., p. 20. 
Dès ses débuts, l'auteure s'est réclamée d'une écriture de la modernité vouée à une certaine entreprise de déconstruction du langage, de remise en question des valeurs sociales rétrogrades, de révision du champ culturel, de refus de la représentation réaliste, bref de rejet des savoirs et des pouvoirs codés d'avance par une élite réactionnaire et répressive. Pour les femmes qui commençaient à écrire, cette gigantesque entreprise se doublait d'une tentative de cerner leur rapport au langage comme dans un désir de Modeler le discours et avoir raison. [...] Chercher fiévreusement la faille, $m^{\prime} y$ glisser et de l' intétrieur déconstruire. Long, laborieux, solitaire. C'est l'éloignement (p. 49). ${ }^{15}$

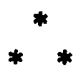

Nous parlerons comme on écrit témoigne d'un renversement des codes narratifs traditionnels dans sa structure même. Le roman se compose de six parties dont deux segments plus longs (I et IV), sans titre, se divisent respectivement en 60 et 61 fragments clairement délimités typographiquement. Gisèle Tuaillon a bien montré dans sa thèse le parallélisme structurel entre les deux ensembles, le recoupement de l'un à l'autre, le rapprochement des sujets traités ainsi que la symétrie des stratégies textuelles ${ }^{16}$. Dans ces deux unités composées de courts textes, rien de linéaire: à peine une ébauche de récit qui s'amorce sur des bribes d'histoire de la narratrice/jeune fille auxquelles font écho des «racontars» de vieille femme intercalés de confidences d'autres femmes, de conversations plus intellectuelles avec une amie et de commentaires de toutes sortes sur la musique, le Mexique, l'enseignement, etc. Rien ne se suit donc, rien ne s'enchaîne, mais Comment est-ce possible de saisir l' enchaînement quand l'élémentaire survie est menacée? (p. 90) Écriture décousue dont le seul fil conducteur demeure la difficile recherche du mot d'une femme sans langage. Le fragment a justement permis à l'auteure du roman d'isoler les scènes, de travailler au montage de la représentation. ${ }^{17}$

À ces deux morceaux brièvement décrits s'ajoutent quatre parties d'une longueur sensiblement égale (de 14 à 17 pages) se donnant d'un trait. Bien que les chapitres III et IV correspondent davantage au récit linéaire, l'écriture s'y présente tout de même éclatée (ainsi que dans les chapitres II et VI). Une syntaxe altérée, une phrase en désagrégation, des scènes confondues, l'ellipse généralisée:

15 L'écriture au féminin a d'ailleurs considérablement contribué au renouvellement des formes et des contenus en littérature. Plusieurs écrivaines québécoises ont privilégié le texte fragmentaire et l'écriture détailliste. N. Schor considère l'esthétique du détail sexuellement marquée et associée au genre féminin:In other words, to focus on the place and function of the detail since the mid-eighteenth century is to become aware the normative aesthetics elaborated and disseminated by the Academy and its members is not sexually neutral; it is an axiology carrying into the field of representation the sexual hierarchies of the phallocentric cultural order. The detail does not occupy a conceptual space beyond the laws of sexual difference: the detail is gendered and doubly gendered as feminine. N. Schor, op. cit., p. 4. Je souligne.

16 Gisèle Tuaillon, op. cit.

17 Gérard Gaudet, loc. cit., p. 114. 
effets d'obscurcissement, signes noués dans une langue trouée qui échappe au sens, à la logique, à l'ordre. Erosion de tous les codes antérieurs (p. 47). Brèche. Sur fond de paroles (p. 36). Ces quatre textes restent donc eux aussi à leurs manière écrits sur le mode fragmental.

Dans un article intitulé «Du fragment au détail», Reda Bensmaïa soutient que le texte fragmentaire constitue la «matrice» de tous les genres, le genre littéraire qui s'autogénère ${ }^{18}$. Pour Barthes, le fragment, c'est l'intermezzo, une pièce intercalée dans un cycle ${ }^{19}$. Toutefois, plus important que le lieu du fragment, est le fait qu'il se génère de l'intérieur, d'une réserve illimitée de «détails» non plus perçus comme des sous-produits ou des échantillons, mais comme le dedans du fragment. Le détail effectue ainsi une sorte de mise en abyme du texte qui le contient, le signifiant du signifiant. Reprenant les distinctions barthésiennes de l'obtus et de l'obvie, Bensmaiia insiste sur l'impossibilité d'assimiler fragment et détail:

Tout d'abord, comme le sens obtus, le rdétail» est discontinu, indifférent d tout récit et d'une manière générale d tout sens «obvie». [...] Mais par rapport au «réel» comme «nature» (instance «réaliste») le "détail » comme usens obtus» du fragment est la marque d' une distance inouïe d̀ tout référent *concret»: il est plutôt «[une] pesée sur le signifiant» $\left[\ldots . .{ }^{20}\right.$

D'où un effet de déplacement, de glissement ou de perte de sens: Un mot mort du réel (p. 84). Détail ou «contre-récit» s'inscrivent sans cesse en dehors de, à côté de, de manière (ex)centrique, à contre-courant.

Une fois la surface du récit explorée, les voix reconnues, les frontières tracées, il faut encore saisir le texte, le décomposer, le désarticuler, le couper en petits morceaux pour que s'éparpillent les «restes» d'écritures disséminés au gré des pages, tous ces «détails» qui s'y multiplient, s'engendrent les uns les autres, s'entrechoquent, s'intervertissent, s'appellent, se parlent.

Nous parlerons comme on écrit en détail(s), ce sont toutes ces histoires banales de dizaines de femmes; ces tableaux/ écrans de lieux désertés; ces corps mutilés, brisés, enchaînés; ces regards baissés, ces oreilles sourdes; ces nouds, ces murs, cette parole étranglée; ce crâne éclaté sur un pavé, cette mémoire perforée; ces voix désolées; cette langue faite de tous les mots, en tous sens (p. 138), ceux de Laure, d'Anaiis, de Virginia et de bien d'autres, tous les sons aussi évidemment. Mots-images qui traversent le récit: une microscopique marche aléatoire (p. 9) d'une jeune femme qui déambule dans les rues de ses villes.

Face d̀ la vie publique, écrit France Théoret, les scènes inacceptables de la vie privée relèvent de faits divers ou de l' anecdote. Or, écrire, c'est rendre public

18 Reda Bensmaīa, loc. cit., p. 356.

19 Roland Barthes par Roland Barthes, op. cit., p. 98.

20 Reda Bensmaïa, loc. cit., p. 367. 
et ça passe par un acte solitaire qui est lié avec le privé. ${ }^{21}$ Le privé ou le particulier ne relève pas du général ou du transcendantal, comme si les drames individuels ne signifiaient que bien peu de choses, Pas même une histoire digne des faits divers. $D u$ tissage quotidien (p. 35). Le roman contient, je l'ai mentionné plus haut, une série de mini-récits racontés sur le ton d'une confidence par une vieille femme ou par la narratrice elle même: histoires de femmes contenues, refoulées dans la mémoire des générations, suite de scènes plus ou moins éclairées selon l'heure du jour et selon le regard de l'auditrice/spectatrice. C'est la dimension du privé qui s'étale en plein jour. Commérages de femmes, sourdes oreilles d' hommes. Les femmes ne sont pas dans l' histoire, elles n'ont que des *histoires», ne font que «des histoires», pour reprendre cette fois les mots de Nicole Brossard et de France Théoret 22 .

Ces histoires créent ce que j'appelle l'effet-musée. Véritable galerie de portraits de femmes dont nous ne percevons bien souvent qu'un trait: un aspect du visage, un geste, une douleur, un soupir, un détail. Sur fond clair-obscur ou sur un grand écran noir et blanc. Effets de montage pour une exposition de dessins «animés» qui ne bougent plus. Toiles statiques en un sens, mais d'un détail à l'autre s'y insinue une forme de mouvement. Le plaisir des sens peutêtre, dans la déchirante nuit de la parole. L'épisode du récit de vie, un temps, une fois entre la naissance et la mort, sorte d'exemplum parfois, dénonce le sort fait aux femmes, à ces femmes: blessures au corps et aux mots. La parole de femme est vide, pur commérage, papotage sans importance, insignifiant. En effet, elles peuvent bien jacasser toutes. Sans importance. Sans conséquence (p. 38), mais Au moins elles disent, elles se dépassent par la parole, elles font qu'arrive au jour cette parole née hors d'elle d partir de laquelle elles mettent au jour tant et tant d'oublis. Elles enroulent sur fond d' une mémoire encagée le mouvement le plus sûr, elles font que germe ce qui n'a de cesse d'être tiré hors la nuit (p. 164).

Faits divers que le viol, la misère, la femme battue, l'ignorance, l'avortement dans la peur, l'abandon, l'accouchement forcé, le meurtre, la mort de ceux qu'on aime? Contes de femmes: historiettes communes et anecdotes sans importance ou jugées telles? Traces, empreintes, histoires ancrées dans la mémoire corporelle. Tatouages sur la peau des filles, marques indélébiles. Des taches sur le papier froissé de la mémoire des générations. La mémoire qui, comme l'écriture, repère, retient, rejette, piétine, tourne en rond, au rythme des répétitions, des successions de motifs, des déplacements du conscient et de l'inconscient. De chaque scène/souvenir, de chaque détail, surgit l'ébauche d'une huile à peindre ou d'un conte à finir.

À ces brefs récits intégrés dans le roman, s'ajoutent d'autres tableaux, le plus souvent topographiques: descriptions succinctes de lieux; cadres incomplets faits d'un objet: d'un angle, d'une perspective, d'un décor, d'une couleur sur

21 France Théoret, «Prendre la parole quand on est femmex, Québec français, $n^{\circ} 47$, octobre 1982 , p. 36.

22 Nicole Brossard et France Théoret, «Préface», la Nef des sorcières, Montréal, Quinze, 1976, p. 8. 
lesquels semble se poser le mouvement lent et circulaire d'une caméra. L'effet photographie domine ici comme dans les exemples suivants:

Le mur, une façade. La rue des briques, escaliers en fer, la parallèle perspective devant derrière au-dessus [...]. (p. 9)

Midi des briques. L'été, la chaleur jaune de la ville. Rue sans arbre. Maisons basses. Aridité. Clarté. Le jour départagé. Bleu du ciel. Façades rouges et brunes, portes de bois dépeintes, fenêtres bigarrées [...]. (p. 77)

Ce sont encore les bidonvilles de Mexico (p. 28), le tableau de Virginia Woolf (p. 46), la cuisine de Louise Valois (p. 86), le collège (p. 95), les peintres mexicains (p. 114), etc. Ces «détails» ou photos/souvenirs agissent en tant qu'omements du fragment: ils ajoutent au texte une dimension nouvelle et en déterminent souvent la couleur, le ton, l'atmosphère. Ils accentuent également l'effet de fragmentation renforcé par l'usage systématique de la phrase courte, sèche, nominale ou adjectivale. Tableaux/entailles dans la chair du texte agrémenté de décors.

Ces épisodes, semblables à des découpures de journaux, tailladent le roman à la manière d'un corps tranché. Les coupures textuelles renvoient au sujet disséqué (je, Ninon, Louise, elle), décentré, fractionné, écartelé entre le désir et la crainte, la survie et la mort, le oui et le non, l'être et le non-être, le sens et le non-sens. La métaphore du corps exemplifie ce décentrement en même temps qu'elle reproduit le processus d'écriture. J'ai la tête inversée, des morceaux de corps me manquent le matin, nous dit la voix centrale du récit. Tout le jour au rapiéçage (p. 62). À la fragmentation du récit correspond le découpage du corps physique: au douloureux effort de ramasser son corps en une pièce répond le difficile travail du raccomodage des mots et du sens:

Quelqu' un écrit comme n'écrivant pas. L'écrit met au jour le processus. Il tend d profiler la pensée par la forme. Si le sens insiste, si au sens se mêle trop de souffrances jusqu'd ne jamais arriver même d la parole répétitive, l' apaisement doit venir d' ailleurs. Car le sens opère exclusivement proche des nerfs à vif. (p. 135)

L'examen des qualificatifs du vocable corps montre nettement la condition éclatée de celle qui parle. En les repérant, j'ai constaté que trois groupes se formaient comme suit (membre, partie du corps, sens sont ici considérés comme variantes du corps): le corps brisé (brisé, fracturé, mutilé, rompu, cassé, traversé, crevé, morcelé, battu, - décomposé, tué, abandonné); le corps dominé (marchandé, impotent, dominé, enfermé, retenu); le corps malade (malade, noyé, vidé, inhabité, ridé, rabougri, asséché, épuisé, affolé, bourré de nourriture, soufflé de lait). Le premier groupe d'épithètes fait ressortir clairement la violence faite au corps (féminin) alors que la deuxième série renvoie davantage à un rapport dominant/ dominé, violence mentale entretenue par le pouvoir en place. Finalement, du 
troisième ensemble se dégage une sorte de conséquence, le corps malade ${ }^{23}$. Les trois derniers termes du premier groupe tendent à s'assimiler à cette dernière catégorie en ce qu'ils suggèrent un aboutissement.

Par ailleurs, les fonctions principales liées aux manifestations du corps se rattachent aux phénomènes d'absorption et de rejet: se remplir, boucher, bourrer, grossir jusqu'à éclater, se remplir de se vider et vomir. De plus, le motif du paquet de chair (p. 12) et de l'obèse revient constamment dans le roman. Entre la boulimie et l'anorexie, le trop-plein et le vide, le surplus et le manque (précisément les deux dimensions du détail mentionnées plus haut). Par ailleurs, extérieurement, la zone du ventre, et intérieurement, l'organe digestif principal, l'estomac, dominent l'imagerie corporelle. Lieu de l'angoisse, l'estomac est un trou béant rouge [qui] s'ouvre et se ferme et engouffre la nécessité de continuer (p. 74). En effet, passée d̀ l'estomac, il n'y a pas de lieu précis, certains jours d'éventrement l'intérieur [me] pousse a l'extérieur (p. 32). Deux pôles donc: la tête (là où ça entre); le ventre (là d'où ça sort). D'un côté le discours, la raison, la pensée, la logique; de l'autre la chair, le désir, le matériel, les émotions. D'où l'éventrement (volontaire) et la fracture du crâne (accidentelle). Le leitmotiv du crâne éclaté sur le pavé traverse le récit et projette le sujet du côté de la déraison; l'éventrement le coupe de ses émotions. Perte de raison, perte de sens.

L'éventrement reprend le thème de l'éclatement du sujet VERS l'intérieur un peu comme la technique détailliste décentre le récit, abolissant l'intrigue, la surface, le sens global, pour l'orienter vers le dedans (du fragment), le sens minimal. Le mouvement ne se fait pas de façon unilatérale: il va dans les deux sens puisque la narratrice se déplace sur un fil qui bouge constamment. En ce sens, dedans/dehors, intérieur/extérieur ne doivent s'entendre que par rapport à un sens qui flotte et non à une position fixe sur deux axes perpendiculaires.

Les paradigmes fixité/mobilité (à rattacher aux composantes binaires lourdeur/légèreté, liquidité/solidité, sommeil/éveil) sont inscrits à plusieurs niveaux dans le texte, mais tout particulièrement dans le motif de la marche ${ }^{24}$. Marcher, écrit Pierre Nepveu, c' est s'exposer aux regards, c'est entrer dans le drame de l'apparaître. ${ }^{25}$ Le lien entre la marche et le regard fait ressortir l'importance de la métaphore de l'œil dans l'œuvre. C'est précisément le rapport au réel et à la représentation de la réalité qui est en jeu dans le discours de l'œil en même temps que la position du sujet face au corps/f(r)iction.

Dans un premier temps, je/elle est l'objet du regard des autres, et même du sien: Pendant des années, je (elle) me voyais me voir (p. 163). Prise dans le

23 Je n'ai retenu pour mon propos que les adjectifs, mais je note en passant que des mentions faites à des formes ou des états de maladie (mentale ou autre) renforcent l'image du corps malade: l'apathie, l'aphasie, la folie, l'invalidité, l'obésité, par exemple.

24 L'importance du motif de la marche dans l'auvre de France Théret a été signalée maintes fois, entre autres par Pierre Nepveu dans aIntérieurs d'une pensée», Estuaire, $n^{\circ} 38,1986$, p. $23-29$. 
regard aliénant, ses yeux sont figés, brisés, baissés, crevés. L'aveuglement, par ailleurs, se situe du côté des générations, des mères, lié au fait de ne pas vouloir voir, de ne pas oser regarder et de s'aveugler, au refus par conséquent. En revanche, la quête d'identité de la narratrice se traduit dans un double mouvement de perception, soit voir et ne pas voir, être là et ne pas y être, habiter et ne pas habiter, simultanément. Par la suite, l'œil s'ouvre complètement: voir, regarder, ouvrir l'œil, avoir à l'œil, yeux ouverts, voyeuse, autant d'affirmations du vu et du vécu de la part du sujet qui entre à la fois dans le champ de vision de l'autre et dans le champ de pensée «moderne». La pratique visuelle pose d'une certaine façon une pratique consciente de l'écriture. De plus, en s'inscrivant dans une vision «éclairée» des lettres, de l'écrit, des choses et des êtres qui l'entourent (réappropriation de la parole), la narratrice/l'écrivaine se situe par rapport à l'épistémologie contemporaine.

Cette dynamique tend à se renverser au moment où le sujet se voit confronté à la fiction de l'existence, au voile des apparences: Certains jours, dit-elle, j'échangerais volontiers des apparences, simulacres du réel pour quelque matière vivante. Dans la trouée, en perspective, leurres sur leurres, d l'infini (p. 169). Aux prises avec des mots et des images, «je» semble coupé de la réalité, toujours voilée, à travers laquelle percent de vagues ombres. Des couches de «faux» sens à traverser: écran, voile, camouflage, semblant, apparence, leurre, masque, décor. Ces termes, dont certains sont abondamment répétés, suggèrent l'idée de la couverture et d'un lieu devant/derrière, dessus (sur)/dessous (sous). La «vérité» de l'objet/mot se cache derrière une vitre teintée. Le critique H.S. Daemmrich a souligné que le détail isolé ne révèle pas, mais voile le réel ${ }^{26}$. La réalité est derrière, le sens est ailleurs, inaccessible; il échappe à l'œil, se dissimule sous des apparences plus ou moins trompeuses, derrière $[. .$.$] des histoires de boule verte,$ écrit France Théoret. La boule verte comme la maladie des nuits abat-jour cachée. Le fil de l' histoire la relie, elle sait qu'il y a la caverne et les ombres. Elle va déposer les surplus (p. 66). Dans la caverne de Platon (trois allusions y sont faites dans le roman), l'essence de la réalité échappe à l'œil humain qui n'y perçoit justement que des ombres. Et si l'écrivaine, assise face au mur de la caveme, se retournait? Apercevrait-elle ces silhouettes qui circulent à l'entrée de la grotte, ou réaliserait-elle qu'elles ne logent, inaccessibles, invisibles, que dans le mur de sa mémoire? Sa mémoire demeurant le seul lieu possible de leur venue au monde, de leur existence. Le lieu du réel, là où des femmes s'entêtent à parler comme elles écrivent. Et tout le reste n'est qu'un effet du réel, une illusion d'optique recréé par des milliers de détails en fête.

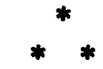

En conclusion, il faut rappeler que le détail interpelle le sens de la vue d'une manière ou d'une autre parce que petit, on le voit à peine, on passe facilement à côté sans le remarquer, mais grossi, élargi, il tend à effacer la toile, le tableau, la 
scène. Autrement dit, on ne lui jette même pas un coup d'œil dans le premier cas alors qu'on n'a d'yeux que pour lui dans le second. Son insignifiance provoque l'oubli du spectateur; son importance crève les yeux, aveugle. Le lecteur/la lectrice sont des voyeurs/voyeuses à la recherche d'un plaisir dit esthétique, et j'ajouterais, vicieux. Si quelquefois le regard précède et suscite le désir du texte, l'inverse est également vrai. Dans tous les cas, une lecture de l'œil est une lecture de détail(s) rejetés ou recherchés.

En fait, le détail modifie profondément le regard que l'on porte sur une œuvre d'art et par extension le statut classique de la lecture et son objet. À ce propos, Bensmaïa ajoute que $C$ 'est dire que pour lire (ou analyser) un tel texte ce n'est plus dे une vision synoptique qu'il faut dès lors faire appel, mais d̀ un «troisième sens», soit à une ECOUTE dont Barthes disait qu'elle était sla métaphore» qui convenait le mieux au «textuel» : c'est-à-dire une lecture de texte, mais comme «orchestration», «contrepoint» et «stéréophonie». ${ }^{27}$ On connaît l'importance de ce demier sens dans le roman de France Theoret (et dans l'ensemble de ses écrits) ${ }^{28}$. Le complexe silence-oreille-musique-voix-cri devrait être repris dans cette perspective. L'analyse de motifs, thèmes et registres contenus dans le lexique du nœud, du mur, de la mémoire, du mouvement et de la marche, de la langue/parole et du geste, éléments qui fonctionnent en «symphonie» dans le récit, permettraient certainement de compléter la lecture amorcée.

Dans Nous parlerons comme on écrit, tout se tient, en fonction d'une unité autre et d'une cohérence liée au chaos et au morcellement de la réalité. Le roman s'élabore en contrepoint basé sur ce que je nomme une esthétique du détail, esthétique que les travaux de Freud d'abord ${ }^{29}$ et, plus près de nous, ceux de Barthes, Foucault, Deleuze, Kristeva, Derrida, pour ne nommer que ceux-là, ont grandement revalorisée. Éparpillées dans le texte, des aquarelles qui ont la couleur des journées chaudes de juin se dessinent. Si la mort se faufile parfois au ras des mots, il y a au détour des lignes des envolées de lumière d'où jaillit la parole. L'encre y coule à flots. Enfin, pourrait-on objecter, ce n'est là qu'un détail. Un détail de poids dans une œuvre si belle et si pleine.

27 Reda Bensmaïa, loc. ciu., p. 370.

28 Voir Claire Lejeune, «Clefs pour une correspondance à quatre voix», Estuaire, $n^{\circ} 38$, 1986, p. 31-39.

29 Voir à ce sujet l'article de K.R. Eisler, *The Function of Details in the Interpretation of Works of Literaturex, The Psychoanalytic Quarterly, janvier 1959, p. 1-20. 